\title{
Purification of molybdenum oxide, growth and characterization of medium size zinc molybdate crystals for the LUMINEU program
}

\author{
V.N. Shlegel ${ }^{1, a}$, L. Berge ${ }^{2}$, R.S. Boiko ${ }^{3}$, M. Chapellier ${ }^{2}$, D.M. Chernyak ${ }^{2,3}$, N. Coron ${ }^{4}$, F.A. Danevich ${ }^{3}$, R. Decourt ${ }^{5}$, \\ V.Ya. Degoda ${ }^{6}$, L. Devoyon ${ }^{7}$, A. Drillien ${ }^{2}$, L. Dumoulin ${ }^{2}$, C. Enss ${ }^{8}$, A. Fleischmann ${ }^{8}$, L. Gastaldo ${ }^{8}$, A. Giuliani ${ }^{2}$, \\ M. Gros ${ }^{7}$, S. Herve ${ }^{7}$, I.M. Ivanov ${ }^{1}$, V.V. Kobychev ${ }^{3}$, Ya.P. Kogut ${ }^{6}$, F. Koskas ${ }^{7}$, M. Loidl ${ }^{7}$, P. Magnier ${ }^{7}$, E.P. Makarov ${ }^{1}$, \\ M. Mancuso ${ }^{2,9}$, P. de Marcillac ${ }^{4}$, S. Marnieros ${ }^{2}$, C. Marrache-Kikuchi ${ }^{2}$, S.G. Nasonov ${ }^{1}$, X.F. Navick ${ }^{7}$, C. Nones ${ }^{7}$, \\ E. Olivieri ${ }^{2}$, B. Paul ${ }^{7}$, Y. Penichot ${ }^{7}$, G. Pessina ${ }^{10}$, O. Plantevin ${ }^{2}$, D.V. Poda ${ }^{2,3}$, T. Redon ${ }^{4}$, M. Rodrigues ${ }^{7}$, O. Strazzer ${ }^{7}$, \\ M. Tenconi ${ }^{2}$, L. Torres ${ }^{4}$, V.I. Tretyak ${ }^{3}$, Ya.V. Vasiliev ${ }^{1}$, M. Velazquez ${ }^{5}$, O. Viraphong ${ }^{5}$ and V.N. Zhdankov ${ }^{11}$ \\ ${ }^{1}$ Nikolaev Institute of Inorganic Chemistry, 630090 Novosibirsk, Russia \\ ${ }^{2}$ Centre de Sciences Nucléaires et de Sciences de la Matière, 91405 Orsay, France \\ ${ }^{3}$ Institute for Nuclear Research, MSP 03680 Kyiv, Ukraine \\ ${ }^{4}$ IAS, Bâtiment 121, UMR 8617 Université Paris-Sud 11/CNRS, 91405 Orsay, France \\ ${ }^{5}$ CNRS, Université de Bordeaux, ICMCB, 87 avenue du Dr. A. Schweitzer, 33608 Pessac cedex, France \\ ${ }^{6}$ Kyiv National Taras Shevchenko University, MSP 03680 Kyiv, Ukraine \\ ${ }^{7}$ CEA-Saclay, F-91191 Gif sur Yvette, France \\ ${ }^{8}$ Institut für Angewandte Physik, Universität Heidelberg, Albert-Ueberle-Strasse 3-5, D-69120 Heidelberg, Germany \\ ${ }^{9}$ Dipartimento di Scienza e Alta Tecnologia dell'Università dell'Insubria, I-22100 Como, Italy \\ ${ }^{10}$ Dipartimento di Fisica dell'Università di Milano-Bicocca e Sezione di Milano Bicocca dell'INFN, Italy \\ ${ }^{11}$ CML Ltd., 630090 Novosibirsk, Russia
}

\begin{abstract}
The LUMINEU program aims at performing a pilot experiment on neutrinoless double beta decay of ${ }^{100} \mathrm{Mo}$ using radiopure $\mathrm{ZnMoO}_{4}$ crystals operated as scintillating bolometers. Growth of high quality radiopure crystals is a complex task, since there are no commercially available molybdenum compounds with the required levels of purity and radioactive contamination. This paper discusses approaches to purify molybdenum and synthesize compound for high quality radiopure $\mathrm{ZnMoO}_{4}$ crystal growth. A combination of a double sublimation (with addition of zinc molybdate) with subsequent recrystallization in aqueous solutions (using zinc molybdate as a collector) was used. Zinc molybdate crystals up to $1.5 \mathrm{~kg}$ were grown by the lowthermal-gradient Czochralski technique, their optical, luminescent, diamagnetic, thermal and bolometric properties were tested.
\end{abstract}

\section{Introduction}

Neutrinoless double beta $(0 \vee 2 \beta)$ decay is a key process in particle physics thanks to its unique ability to test the Majorana nature of neutrino and lepton number conservation, the absolute scale and the hierarchy of neutrino mass [1, 2, 3, 4]. Low temperature scintillating bolometers are considered as extremely promising detectors to search for $0 v 2 \beta$ decay in different nuclei [5, $6,7,8,9,10,11]$. Recently developed technique to grow large high quality radiopure zinc molybdate $\left(\mathrm{ZnMoO}_{4}\right)$ crystal scintillators $[9,10,12,13,14,15]$ makes this material advantageous for low temperature bolometric experiments to search for $0 v 2 \beta$ decay of ${ }^{100}$ Mo. Here we report further progress in deep purification of molybdenum and growth of $\mathrm{ZnMoO}_{4}$ crystals. First results of the crystals characterization are presented too.

\section{Production of $\mathrm{ZnMoO}_{4}$ crystals}

\subsection{Purification of molybdenum}

High purity molybdenum and zinc are required to grow high quality radiopure $\mathrm{ZnMoO}_{4}$ crystal scintillators. While a high purity zinc oxide is commercially available, molybdenum should be additionally purified. Furthermore, there are no commercially available molybdenum compounds that are tested for the presence of radioactive elements and have the required level of radioactive contamination. Such a test of the raw materials for crystal growth is extremely difficult and requires long measurement procedure. Typically high sensitivity radiopurity tests can be only done after crystal

\footnotetext{
${ }^{\mathrm{a}}$ Corresponding author: shlegel@ niic.nsc.ru
} 
growth using calorimetric method. Moreover, development of efficient purification methods with minimal losses of molybdenum is strongly required to produce $\mathrm{ZnMoO}_{4}$ crystals from enriched molybdenum, whose contamination is typically on the level of tens hundreds ppm [16] (keeping also in mind the necessity to recycle the costly enriched material). We have developed a two stages technique of molybdenum purification consisting of sublimation of molybdenum oxide in vacuum (with addition of zinc molybdate) and double recrystallization from aqueous solutions by coprecipitation of impurities on zinc molybdate sediment.

\subsubsection{Purification of $\mathrm{MoO}_{3}$ by sublimation}

Sublimation of molybdenum oxide under atmospheric pressure with subsequent leaching in aqueous solutions with ammonia is widely used in the industry of molybdenum. Nevertheless the concentration of impurities, particularly of tungsten (on the level of up to $0.5 \mathrm{wt} \%$ even in the high purity grade materials) still exceeds the $\mathrm{ZnMoO}_{4}$ crystal growth requirements. Even additional vacuum sublimation of molybdenum oxide proved to be insufficient. According to [17] separation of tungsten and molybdenum is a well known problem. Besides, the sublimation of $\mathrm{MoO}_{3}$ is not efficient enough to reduce traces e.g. of $\mathrm{Ca}, \mathrm{Na}$ and $\mathrm{Si}$ to the level below $20-70$ ppm.

We have assumed that during sublimation at high temperature the following exchange reaction could occur:

$$
\mathrm{ZnMoO}_{4}+\mathrm{WO}_{3}=\mathrm{ZnWO}_{4}+\mathrm{MoO}_{3} \uparrow .
$$

Such a reaction should reduce the concentration of tungsten, and therefore can be used for separation of molybdenum from tungsten. To prove this possibility, we have prepared a sample of $\mathrm{MoO}_{3}$ powder with $10 \mathrm{wt} \%$ of $\mathrm{WO}_{3}$. The concentration of tungsten in the $\mathrm{MoO}_{3}$ product after sublimation was reduced to $0.1 \mathrm{wt} \%$.

One more confirmation of the method's efficiency was obtained by chemical and X-ray diffraction analysis of the rests after the sublimations performed with an aim to purify molybdenum for crystal growth (the amount of the rests is typically $1-3 \mathrm{wt} \%$ of the initial amount of the purified material). The bottoms after a few sublimation processes were mixed and annealed in the air atmosphere to oxidize residues of metals. Then we have carried out sublimation of the sample in vacuum to reduce presence of $\mathrm{MoO}_{3}$. Atomic emission analysis, performed in the analytical laboratory of the Nikolaev Institute of Inorganic Chemistry, gives the following elemental composition of the bottoms: $\mathrm{Ca}-0.14 \mathrm{wt} \% ; \mathrm{Cu}$ - 0.011wt $\% ; \mathrm{Fe}-0.064 \mathrm{wt} \% ; \mathrm{K}-1 \mathrm{wt} \% ; \mathrm{Mg}-$ $0.026 \mathrm{wt} \% ; \mathrm{Na}-0.13 \mathrm{wt} \% ; \mathrm{Si}-2.6 \mathrm{wt} \% ; \mathrm{Mo}-22 \mathrm{wt} \%$; W - 18wt $\%$; $\mathrm{Zn}-14 \mathrm{wt} \%$. Oxides of molybdenum and silicon, tungstate (in form of tungstate-molybdate) and molybdate of zinc, as well as $\mathrm{K}_{2} \mathrm{Mo}_{7} \mathrm{O}_{22}$ and $\mathrm{K}_{2} \mathrm{MgSi}_{5} \mathrm{O}_{12}$, have been identified in the bottoms with the help of $\mathrm{X}$-ray diffraction analysis. At the same time, tungsten oxide (present in the initial product) was not detected in the bottoms. The data supported occurring of the exchange reaction (1) and confirmed efficiency of molybdenum oxide sublimation in vacuum with addition of zinc molybdate.

To purify molybdenum for $\mathrm{ZnMoO}_{4}$ crystal growth, we have added up to $1 \%$ of high purity zinc molybdenum (obtained earlier in the course of the R\&D) to the $\mathrm{MoO}_{3}$ prepared for sublimation. The obtained sublimates contained mixture of molybdenum oxides of different composition and color, which hinders their use for $\mathrm{ZnMoO}_{4}$ synthesis. The sublimates were then annealed in the air atmosphere to obtain yellow color stoichiometric $\mathrm{MoO}_{3}$. The sublimates were analyzed by atomic emission spectrometry. The results are presented in Table 1. One can see that the purity level of $\mathrm{MoO}_{3}$ was improved one-two orders of magnitude after the double sublimation process. The sublimation also should remove metal oxides, which have a high vapor pressure at temperatures up to a thousand degrees.

Table 1. Efficiency of molybdenum oxide purification by sublimation.

\begin{tabular}{|l|c|c|c|c|}
\hline \multirow{2}{*}{ Material } & \multicolumn{4}{|c|}{ Concentration of impurities (ppm) } \\
\cline { 2 - 5 } & Si & K & Fe & W \\
\hline $\begin{array}{l}\text { Initial } \\
\mathrm{MoO}_{3}\end{array}$ & 600 & $100-500$ & 6 & $200-500$ \\
\hline $\begin{array}{l}\text { After 1st } \\
\text { sublima- } \\
\text { tion }\end{array}$ & $100-500$ & $10-50$ & $2-6$ & $100-200$ \\
\hline $\begin{array}{l}\text { After 2nd } \\
\text { sublima- } \\
\text { tion }\end{array}$ & 70 & $1-8$ & $<1$ & $30-40$ \\
\hline
\end{tabular}

\subsubsection{Purification by recrystallization from aqueous} solutions

Finally the molybdenum was purified by double recrystallization of ammonium molybdate in aqueous solutions with the deposition of impurities on zinc molybdate sediment. For this purpose molybdenum oxide was dissolved in solution of ammonia. Mono-molybdates and various poly-compounds and hetero-poly compounds are formed depending on the mixing ratio of the components. A composition of the compounds depends on the acidity of the solution and components concentration. Molybdates in aqueous solutions form normal molybdate:

$$
\mathrm{MoO}_{3}+2 \mathrm{NH}_{4} \mathrm{OH}=\left(\mathrm{NH}_{4}\right)_{2} \mathrm{MoO}_{4}+\mathrm{H}_{2} \mathrm{O}
$$

and ammonium hepta-molybdates, $\left(\mathrm{NH}_{4}\right)_{\mathrm{x}} \mathrm{H}_{(6-\mathrm{x})}\left[\mathrm{Mo}_{7} \mathrm{O}_{24}\right]$. Poly-molybdates can form, in presence of impurities, during long time exposure, ammonium salts of silicomolybdic and phosphor-molybdic acids, for instance $\mathrm{H}_{8}\left[\mathrm{Si}\left(\mathrm{Mo}_{2} \mathrm{O}_{7}\right)_{6}\right], \mathrm{H}_{7}\left[\mathrm{P}\left(\mathrm{Mo}_{2} \mathrm{O}_{7}\right)_{6}\right]$. Role of the central atom, except $\mathrm{Si}(\mathrm{IV})$ and $\mathrm{P}(\mathrm{V})$, can perform also $\mathrm{V}(\mathrm{V}), \mathrm{Ge}(\mathrm{IV})$, $\mathrm{Cr}(\mathrm{III})$, etc., while ligands of the inner sphere can be ions $\mathrm{WO}_{4}{ }^{2-}, \quad \mathrm{VO}^{3-}, \mathrm{CrO}_{4}{ }^{2-}, \mathrm{TeO}_{4}{ }^{2-}$. Solutions of polymolybdates at $\mathrm{pH}<6$ are able to dissolve oxides and hydroxides of many metals, e.g. $\mathrm{ZnO}, \mathrm{Fe}(\mathrm{OH})_{3}, \mathrm{Ni}(\mathrm{OH})_{2}$, $\mathrm{Cu}(\mathrm{OH})_{2}$, etc. As a result, a partial co-crystallization of impurities could occur. Thus, recrystallization of ammonium para-molybdate is not efficient enough for molybdenum purification. Besides, a typical concen- 
Table 2. Purity level of $\mathrm{MoO}_{3}$ before and after purification by recrystallization and sublimation. Data for commercial 5N5 grade product, and enriched ${ }^{100} \mathrm{Mo}$ material are given for comparison.

\begin{tabular}{|l|c|c|c|c|c|c|c|c|}
\hline \multicolumn{1}{|c|}{ Material } & \multicolumn{9}{c|}{ Concentration of impurities (ppm) } \\
\cline { 2 - 9 } & $\mathbf{N a}$ & $\mathbf{M g}$ & $\mathbf{S i}$ & $\mathbf{K}$ & $\mathbf{C a}$ & $\mathbf{F e}$ & $\mathbf{Z n}$ & $\mathbf{W}$ \\
\hline Initial $\mathrm{MoO}_{3}$ & 60 & 1 & 60 & 50 & 60 & 8 & 10 & 200 \\
\hline Recrystallization from aqueous solutions & 30 & $<1$ & 30 & 20 & 40 & 6 & 1000 & 220 \\
\hline $\begin{array}{l}\text { Sublimation and recrystallization from } \\
\text { aqueous solutions }\end{array}$ & - & $<1$ & 30 & 10 & 12 & 5 & 500 & 130 \\
\hline $\begin{array}{l}\text { Double sublimation and recrystallization } \\
\text { from aqueous solutions }\end{array}$ & - & $<1$ & - & $<10$ & $<10$ & $<5$ & 70 & $<50$ \\
\hline $\begin{array}{l}\text { 5N5 grade } \mathrm{MoO}_{3} \text { used to produce ZnMoO } \\
\text { crystal studied in [12, 26] }\end{array}$ & 24 & - & 9 & 67 & 15 & $<18$ & - & 96 \\
\hline $\begin{array}{l}\text { Samples of enriched isotope }{ }^{100} \text { Mo used in } \\
{[16] \text { (before purification, data of producer) }}\end{array}$ & 10 & $<10$ & $50-360$ & $<30$ & $40-50$ & $10-80$ & - & 200 \\
\hline
\end{tabular}

tration of impurities in high purity commercial $\mathrm{MoO}_{3}$ is relatively low $(1-100 \mathrm{ppm})$. As a result, the impurity sediments appear in a form of fine microcrystals, hardly removable by filtration.

To improve efficiency of the recrystallization process, we have used zinc oxide to initiate precipitation (taking into account that zinc does not affect the crystal quality). $\mathrm{ZnO}$ on the level of $1-2 \mathrm{~g} / \mathrm{L}$ was dissolved in the ammonium para-molybdate solution at $\mathrm{pH}>6$, then ammonia was added to the solution to reach $\mathrm{pH}=7-8$. After several hours of exposure precipitation of zinc molybdate occurs. The $\mathrm{ZnMoO}_{4}$ sediment sorbs impurities from the solution. Further increasing of $\mathrm{pH}$ leads to precipitation of contaminants in the form of hydroxides. It should be stressed, the basic solution with $\mathrm{pH} \approx 8-9$ provides the most favorable conditions for thorium and uranium precipitation. After separation of the sediment, the solution was evaporated to $70 \%$. Then ammonium oxalate was added to the solution to bind the residues of iron impurities. Results of the purification are presented in Table 2 .

It should be also stressed that using of the additional "wet" chemistry procedure is also encouraged by the fact that large crystal grains of $\mathrm{MoO}_{3}$ are formed in sublimation process, which provides certain difficulties to produce radiopure $\mathrm{ZnMoO}_{4}$ powder (an additional procedure of the oxide grains grinding could contaminate the material). Subsequent dissolution of the molybdenum oxide in ammonia allows to obtain high purity $\mathrm{MoO}_{3}$ perfectly fine for further synthesis of $\mathrm{ZnMoO}_{4}$ powder.

The molybdenum oxide purified by twice recrystallization procedure from aqueous solutions and high purity grade $\mathrm{ZnO}$ produced by Umicore were used to synthesize $\mathrm{ZnMoO}_{4}$ powder for crystal growth.

\section{$2.2 \mathrm{ZnMoO}_{4}$ crystal growth}

Several $\mathrm{ZnMoO}_{4}$ crystal boules were grown in air atmosphere from the purified input powder by the lowthermal-gradient Czochralski technique [18, 19, 20] in platinum crucibles $\varnothing 40$ and $\varnothing 80 \mathrm{~mm}$ (it should be mentioned that, according to the certificates of the platinum crucibles, iron content in the platinum does not exceed $40 \mathrm{ppm})$. The temperature gradient was kept below $1 \mathrm{~K} / \mathrm{cm}$, the rotational speed was in the range of
$5-20$ rotations per minute with the crystallization rate of $0.8-1.2 \mathrm{~mm} /$ hour. A low crystallization rate was kept during growing of upper cone of the crystal boules. Rotational speed was decreased from the start to the end of the growth process in $1.5-2$ times. The yield of the produced boules was on the level of $80 \%$, which is an important achievement taking into account the future plans to produce crystals from enriched ${ }^{100} \mathrm{Mo}$. Four optical elements (two $\varnothing 20 \times 40 \mathrm{~mm}$ and two $\varnothing 35 \times 40$ $\mathrm{mm}$ with masses $55 \mathrm{~g}$ and $160 \mathrm{~g}$, respectively) were cut and polished for low temperature measurements. Several small samples were produced for optical, luminescent, diamagnetic and thermal tests.

\section{Characterization of $\mathrm{ZnMoO}_{4}$ crystals}

\subsection{Optical absorption}

Visible and near infrared absorption spectra of $\mathrm{ZnMoO}_{4}$ crystal were recorded with a Varian Cary 5000 spectrophotometer. The transmission coefficient, $\mathrm{T}$, was measured on a $2.0 \mathrm{~mm}$-thick single crystal and found to be higher than 0.5 from $327 \mathrm{~nm}$ to $4.96 \mu \mathrm{m}$. The absorption coefficient was calculated as $\alpha=-\log \mathrm{T} \times \ln 10 / t$, with $t$ the thickness of the crystal. The data are presented in Fig. 1.

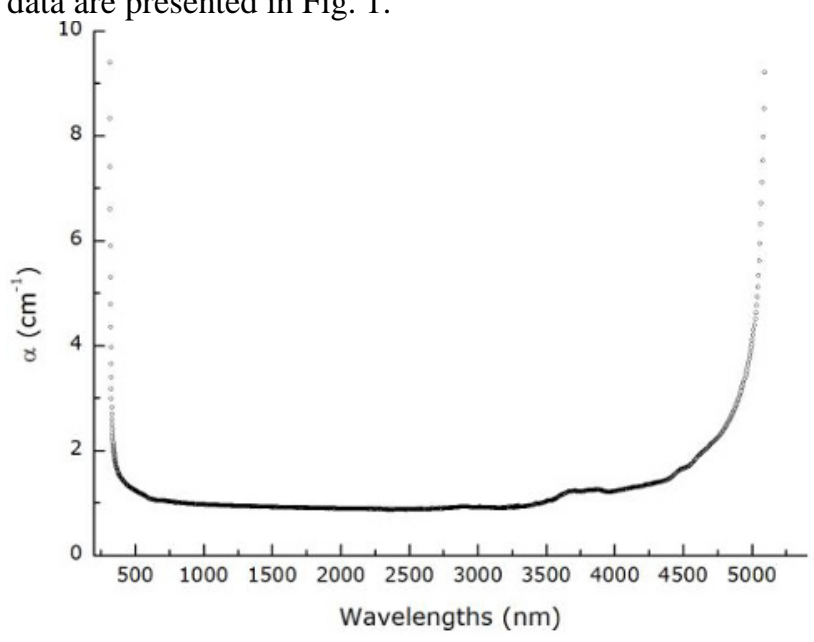

Figure 1. Absorption of $2 \mathrm{~mm}$ thick $\mathrm{ZnMoO}_{4}$ single crystal. $\alpha$ denotes absorption coefficient. 
The cut-off wavelengths are $313 \mathrm{~nm}(\approx 3.96 \mathrm{eV})$ and $5.13 \mu \mathrm{m}$. The absorption coefficient decreases from 1.47 to $0.89 \mathrm{~cm}^{-1}$ in the wavelength region from $400 \mathrm{~nm}$ to 2 $\mu \mathrm{m}$, but there does not seem to have a broad absorption band around $440 \mathrm{~nm}$ that could be ascribed to $\mathrm{Fe}^{2+} / \mathrm{Fe}^{3+}$ impurities as described in [21] and [22]. This is due to the low Fe concentration in the crystal $\left(\approx 1.71 \times 10^{16} \mathrm{~cm}^{-3}\right)$ and in fact, it can be stated that a safe detection limit around $440 \mathrm{~nm}$ by such transmission experiments is $\sim 10^{18}$ atoms of Fe per $\mathrm{cm}^{3}$. Such a low absorption coefficient turns out to be lower than that of the orange crystals grown in [23] and [24], which exhibit $\alpha_{\text {abs }}(<550 \mathrm{~nm}) \geq 2.5 \mathrm{~cm}^{-1}$. The refractive index at $650 \mathrm{~nm}$, obtained from $1+\left(1-\mathrm{T}^{2}\right)^{1 / 2} / \mathrm{T}$ (no Fresnel losses), is $\approx 1.96$, close to the value $\sim 1.91$ given in [25] and to the values $1.87-2.01$ (taking into account biaxiality of the material) obtained in [15] for the wavelengths $406-655 \mathrm{~nm}$.

\subsection{Luminescence under X-ray excitation}

The luminescence of the $\mathrm{ZnMoO}_{4}$ crystal sample $(10 \times 10 \times 2 \mathrm{~mm})$ was investigated as a function of temperature between 8 and $290 \mathrm{~K}$ under X-ray excitation. The sample was irradiated by X-rays from a BHV7 tube with a rhenium anode $(20 \mathrm{kV}, 20 \mathrm{~mA})$. Light from the crystal was detected in the visible region by a FEU-106 photomultiplier (sensitive in the wide wavelength region of 300-800 nm) and in the near infrared region by FEU83 photomultiplier with enhanced sensitivity up to $\approx 1$ $\mu \mathrm{m}$. Spectral measurements were carried out using a high-aperture MDR-2 monochromator. Emission spectra measured at 8, 118 and $290 \mathrm{~K}$ are shown in Fig. 2. A broad band in the visible region with a maximum at 550 $\mathrm{nm}$ was observed at room temperature. At $8 \mathrm{~K}$ luminescence exhibits an emission band with a maximum at $\approx 600 \mathrm{~nm}$ in agreement with the results of previous studies $[15,23,26]$. We have also observed a band at approximately $480 \mathrm{~nm}$ occurring below the liquid nitrogen temperature.

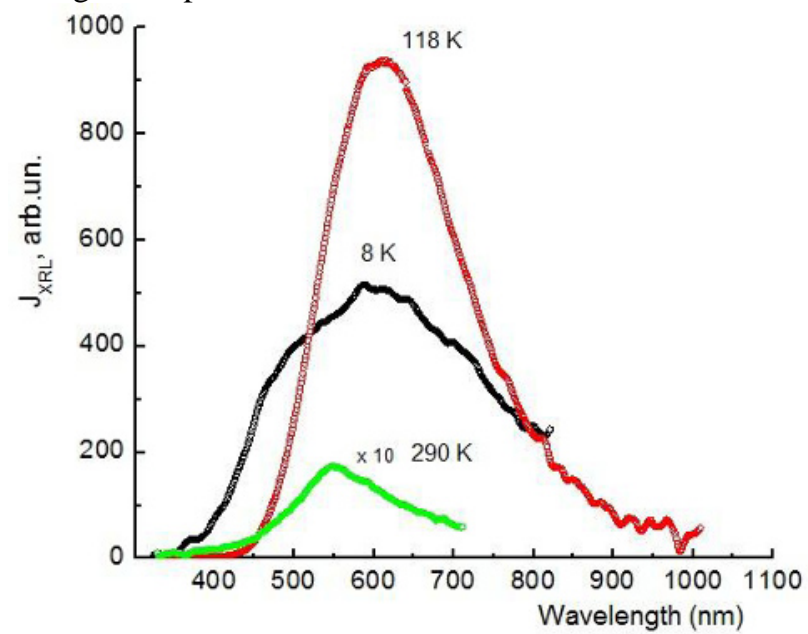

Figure 2. Emission spectra of $\mathrm{ZnMoO}_{4}$ crystal under X-ray excitation at the temperatures 290, 118 and $8 \mathrm{~K}$. $\mathbf{J}_{\mathrm{XRL}}$ denotes intensity of luminescence.

\subsection{Magnetic susceptibility}

Magnetic susceptibility was measured using a Quantum Design SQUID MPMS XL magnetometer operating in the $4.2-350 \mathrm{~K}$ temperature range and in the $0-5 \mathrm{~T}$ magnetic field range. The crystal mass was $210 \mathrm{mg}$ and its volumic mass assumed to be $4.19 \mathrm{~g} / \mathrm{cm}^{3}$. It was mounted in a capsule placed in a straw and the negligibly small diamagnetic contribution $\left(|\chi|<10^{-7}\right)$ of the capsule was not subtracted from our data. The $\mathrm{ZnMoO}_{4}$ proved to be weakly diamagnetic with a MKSA $\chi=-(8.0 \pm 0.2) \times 10^{-6}$ over the whole temperature range investigated, from 20 to $320 \mathrm{~K}$. Thus, paramagnetic impurities such as $\mathrm{Fe}^{2+}$ or $\mathrm{Fe}^{3+}$ could not be evidenced even under higher applied magnetic fields up to $0.2 \mathrm{~T}$.

\subsection{Specific heat measurements}

Specific heat measurements were made on a $3 \times 3 \times 2 \mathrm{~mm}^{3}$ single crystal to optimize the exchange surface and avoid too much thermal inertia. The crystal was fixed on a sapphire sample holder with vacuum grease. The sample holder was mounted on the measurement shaft of a Quantum Design PPMS equipment interfaced to operate with a $2-\tau$ pulse-step method corrected for the grease baseline. The results of the measurements are presented in Fig. 3. The phononic contribution could be approximated for temperatures higher than $\sim 23 \mathrm{~K}$ by means of high-temperature series expansion:

$$
\mathrm{C}_{p, p h .} \propto 1+\sum_{i=1}^{4} B_{i}\left[1+\left(2 \pi \frac{T}{\theta_{D}}\right)^{2}\right]^{-i}
$$

(formula (5) from [27], and red curve in Fig. 3), which yielded a high Debye temperature of $\approx 625.1 \mathrm{~K}$ and the following Bernoulli numbers: $\mathrm{B}_{1}=1.9091, \mathrm{~B}_{2}=1.86714$, $\mathrm{B}_{3}=-0.96009, \mathrm{~B}_{4}=-0.00907$. No long range order (LRO) effect was observed down to $4 \mathrm{~K}$. The $\mathrm{C}_{\mathrm{p}} / 3 \mathrm{NR}$ ratio at $351 \mathrm{~K}$ reaches 0.87 and remains lower than the Dulong and Petit limit, which suggests low anharmonic effects at play at this temperature, consistent with the high Debye temperature obtained by the HTS fit.

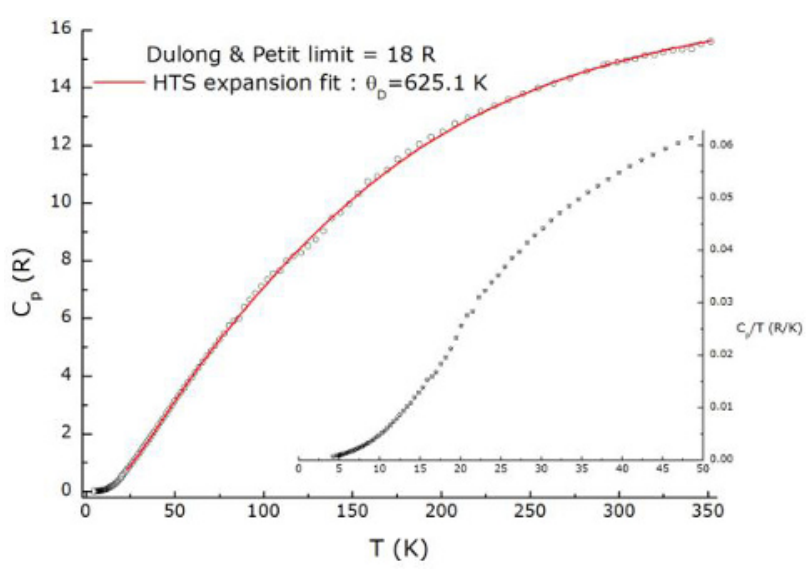

Figure 3. Specific heat versus temperature of a $\mathrm{ZnMoO}_{4}$ single crystal. The inset shows $\mathrm{C}_{\mathrm{p}} / \mathrm{T}$ vs $\mathrm{T}$ at low temperature to evidence the absence of any LRO down to $4 \mathrm{~K}$. 


\subsection{Low temperature tests}

The operation of the scintillating bolometers with the produced $\mathrm{ZnMoO}_{4}$ crystals at low temperatures was performed in the cryogenic laboratories of the CSNSM (Orsay). Two $\mathrm{ZnMoO}_{4}$ samples $(55 \mathrm{~g}$ and $160 \mathrm{~g}$ ) were installed in a high-power dilution refrigerator with a large experimental space. A single photodetector, consisting of a 2" Ge disk and instrumented with a Neutron Transmutation Doped Ge thermistor as a temperature sensor, identical to those attached at the $\mathrm{ZnMoO}_{4}$ crystals, collected the scintillation light emitted by both samples (see Fig. 4).

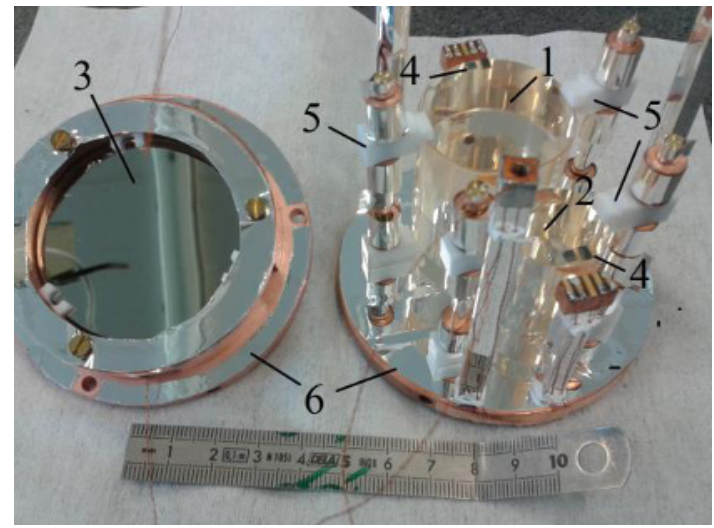

Figure 4. Photograph of the detectors setup: (1) $\mathrm{ZnMoO}_{4}$ crystal $\varnothing 35 \times 40 \mathrm{~mm}$; (2) $\mathrm{ZnMoO}_{4}$ crystal $\varnothing 20 \times 40 \mathrm{~mm}$; (3) Ge slab (photodetector); (4) NTD thermistors; (5) PTFE supporting elements; (6) Copper support of the detector covered by light reflector foil.

The copper heat-sink temperature was stabilized at 18 $\mathrm{mK}$, and the detector operation temperature was about 1 $\mathrm{mK}$ higher due to sensor biasing. Both samples and light detector performed well, with an excellent signal-to-noise ratio. Unfortunately, aboveground operation is marginally compatible with such large crystals. In the large detector, practically every time window containing a full pulse contains also other pulses. One can see the pile-up effect on Inset of Fig. 5 where 4 seconds streaming data accumulated with a weak ${ }^{232} \mathrm{Th}$ gamma source are shown. It should be stressed, the pile-up remains substantial also for the background data acquired without calibration source. This affects the energy resolution of the detector, which is expected to be much better in underground operation under heavy shielding with much lower pile-up effect.

In spite of that, a preliminary useful characterization can be performed in terms of signal amplitude, light yield, light quenching factors for alpha particles and crystal radiopurity. The results for the two crystals are summarized in Table 3. The difference in thermal response is due to the intrinsic irreproducibility of the thermal coupling in this type of detectors. The values of the quenching factor and of the light yield are perfectly compatible with those reported in the literature. Fig. 5 shows a ${ }^{232}$ Th calibration and Fig. 6 presents a light-heat scatter plot accumulated with the large $\mathrm{ZnMoO}_{4}$ crystal. In terms of radiopurity, we will give quantitative estimations in a work in preparation. We can anticipate however that, with the exception of the ${ }^{210}$ Po line at 5.41
$\mathrm{MeV}$, no internal alpha line emerged in the energy spectrum after about two weeks of data taking.

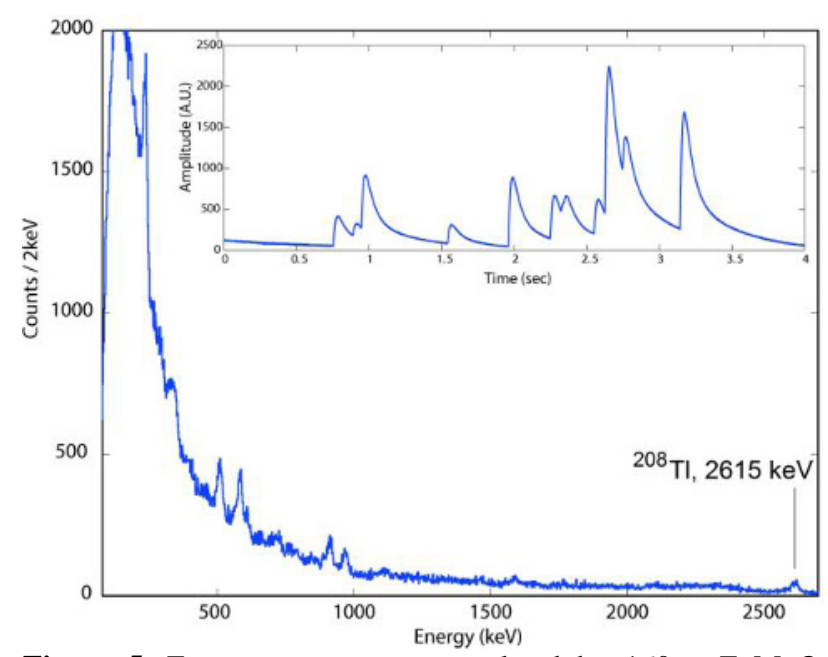

Figure 5. Energy spectrum accumulated by $160 \mathrm{~g} \mathrm{ZnMoO}_{4}$ bolometer with ${ }^{232} \mathrm{Th} \gamma$ source. (Inset) Typical pile-up effect due to a slow time response of the bolometric detector.

Table 3. Performance of $55 \mathrm{~g}$ and $160 \mathrm{~g} \mathrm{ZnMoO}_{4}$ detectors. QF denote quenching factor of $\alpha$ particle signals with respect to $\beta$ particle signals for the same deposited energy $(\sim 5.4 \mathrm{MeV})$.

\begin{tabular}{|l|c|c|}
\hline \multicolumn{1}{|c|}{ Parameter } & $\mathbf{5 5} \mathbf{g}$ & $\mathbf{1 6 0} \mathbf{g}$ \\
\hline Light yield $(\mathrm{keV} / \mathrm{MeV})$ & 0.98 & 0.96 \\
\hline QF & 0.153 & 0.156 \\
\hline
\end{tabular}

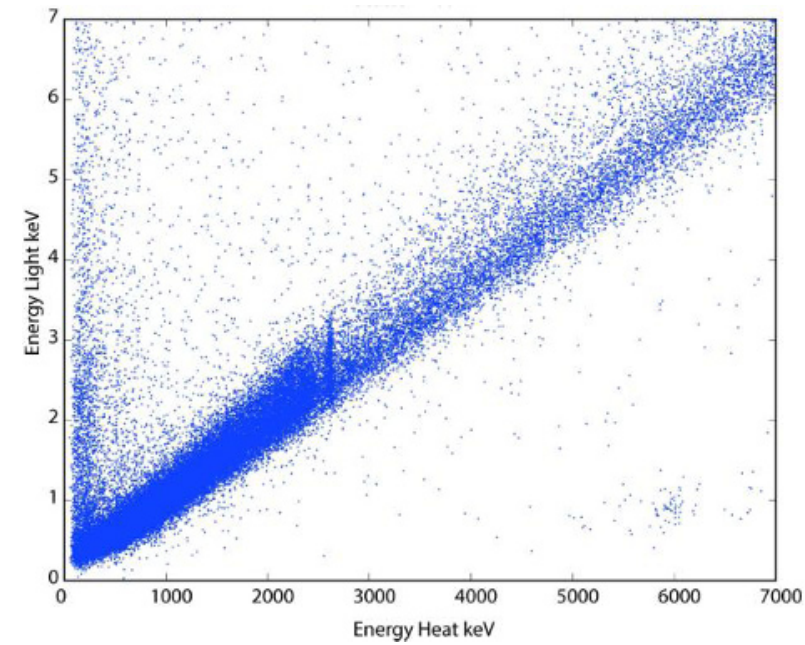

Figure 6. The scatter plot of light and heat signals for $160 \mathrm{~g}$ $\mathrm{ZnMoO}_{4}$ crystal accumulated over $81 \mathrm{~h}$ in aboveground set-up in Orsay. The $\alpha$ band (populated mainly by ${ }^{210} \mathrm{Po}$ internal contamination of the crystal) is neatly separated from the $\beta$ band (containing also $\gamma$ quanta and cosmic muons).

\section{Conclusions}

The LUMINEU program aims at performing a pilot experiment on neutrinoless double beta decay of ${ }^{100} \mathrm{Mo}$ using radiopure $\mathrm{ZnMoO}_{4}$ crystals operated as scintillating bolometers. This problem requires development of methods of molybdenum purification to obtain crystals with desired characteristics. 
Different approaches of molybdenum purification for $\mathrm{ZnMoO}_{4}$ crystals growth were elaborated. A purification using two stages sublimation (with addition of zinc molybdate) and recrystallization from aqueous solutions of ammonium para-molybdate (using zinc molybdate as a collector) is a promising approach to purify molybdenum for high quality radiopure $\mathrm{ZnMoO}_{4}$ crystals growth.

A first batch of LUMINEU crystals with mass up to $1.5 \mathrm{~kg}$ have been successfully grown by by the lowthermal-gradient Czochralski technique, and their optical, luminescent, diamagnetic, thermal and bolometric properties were tested. Characterization of the material is in progress.

In the future, crystals of increasing mass from deep purified precursors will be developed for the LUMINEU experiment, including crystals enriched in the isotope ${ }^{100} \mathrm{Mo}$.

\section{Acknowledgments}

The development of $\mathrm{ZnMoO}_{4}$ scintillating bolometers is part of the LUMINEU program (Luminescent Underground Molybdenum Investigation for NEUtrino mass and nature), a project receiving funds from the L'Agence nationale de la recherche (France). The work was supported in part by the project "Cryogenic detector to search for neutrinoless double beta decay of molybdenum" in the framework of the Programme "Dnipro" based on Ukraine-France Agreement on Cultural, Scientific and Technological Cooperation.

\section{References}

1. W. Rodejohann, Int. J. Mod. Phys. E 20, 1833 (2011)

2. S.R. Elliott, Mod. Phys. Lett. A 27, 1230009 (2012)

3. J.D. Vergados, H. Ejiri, F. Simkovic, Rep. Prog. Phys. 75, 106301 (2012)

4. A. Giuliani and A. Poves, Adv. High En. Phys. 2012, 857016 (2012)

5. S. Pirro et al., Phys. At. Nucl. 69, 2109 (2006)

6. A. Giuliani, J. Low Temp. Phys. 167, 991 (2012)

7. C. Arnaboldi et al., Astropart. Phys. 34, 143 (2010)

8. J. Lee et al., Astropart. Phys. 34, 732 (2011)

9. J.W. Beeman et al., Phys. Lett. B 710, 318 (2012)

10. J.W. Beeman et al., Astropart. Phys. 35, 813 (2012)

11. J.W. Beeman et al., JINST 8, P05021 (2013)

12. L. Gironi et al., JINST 5, P11007 (2010)

13. J.W. Beeman et al., J. Low Temp. Phys. 167, 1021 (2012)

14. J.W. Beeman et al., Eur. Phys. J. C 72, 2142 (2012)

15. D.M. Chernyak et al., Nucl. Instr. Meth. A 729, 856 (2013)

16. P. Belli et al., Nucl. Phys. A 846, 143 (2010)

17. A.N. Zelikman, B.G. Korshunov, Metallurgy of Rare Metals (Metallurgy, Moscow, 1991, in Russian)

18. A.A. Pavlyuk et al., Proc. of the APSAM-92, Asia Pacific Society for Advanced Materials, Shanghai, 26-29 April 1992, Institute of Materials Research, Tohoku University, Sendai, Japan, 1993, p. 164.
19. Yu.A. Borovlev et al., J. Cryst. Growth 229, 305 (2001)

20. E.N. Galashov et al., Functional Materials 17, 504 (2010)

21. D. Spassky et al., Phys. Stat. Sol. A 206, 1579 (2009)

22. A.M. Dubovik et al., Acta Phys. Pol. A 117, 15 (2010)

23. L.I. Ivleva et al., Crystallog. Rep. 53, 1087 (2008) [Kristallografiya 53, 1145 (2008)]

24. L.L. Nagornaya et al., Functional Materials 16, 54 (2009)

25. L.G. Van Uitert et al., J. Amer. Ceram. Soc. 46, 512 (1963)

26. L.L. Nagornaya et al., IEEE Trans. Nucl. Sci. 56, 2513 (2009)

27. J.E. Gordon et al., Sol. St. Comm. 69, 625 (1989) 